\title{
Consumo hídrico e desempenho de Kalanchoe cultivado em substratos alternativos
}

\author{
Water absorption and performance of the Kalanchoe grown in alternative substrates
}

\author{
Angélica Rossana Castro de SouzaI ${ }^{\mathrm{I}}$ Marcia Xavier PeiterII Adroaldo Dias Robaina II \\ Fátima Cibele Soares ${ }^{\text {III }}$ Ana Rita Costenaro Parizi ${ }^{\text {III }}$ Rafael Camargo Ferraz ${ }^{\text {I }}$
}

\section{RESUMO}

Com o crescente consumo de flores e plantas ornamentais de vaso, a busca por elementos alternativos para substratos torna-se necessária, a fim de reduzir os custos da produção. Além disso, é importante quantificar as características físico-hídricas de forma a estabelecer o calendário de irrigações com base nesses materiais. A partir disso, este trabalho teve por objetivos avaliar o desempenho $e$ o consumo de água de Kalanchoe blossfeldiana Poelln, cultivar 'Gold Jewel', em 10 substratos alternativos, e definir os mais indicados para a produção comercial nas condições ambientais da região centro-oeste do Estado do Rio Grande do Sul. Foram testados substratos compostos por diferentes proporções de casca de arroz carbonizada, cinzas de casca de arroz, matéria orgânica doméstica decomposta, esterco bovino e esterco de aves. O experimento foi conduzido em casa de vegetação, em delineamento experimental inteiramente casualizado, com quatro repetições. No final do ciclo, foram avaliados: altura, número de folhas, número de inflorescências, número de flores e diâmetro da copa das plantas nos diferentes tratamentos. Por meio dos resultados, pode-se concluir que, para produção de plantas, os substratos mais indicados são $100 \%$ em volume de matéria orgânica e $50 \%$ em volume de matéria orgânica $+50 \%$ em volume de esterco bovino.

Palavras-chave: irrigação, balanço hídrico, flor-da-fortuna.

\section{ABSTRACT}

With the increase in the flowers and ornamental crops consumption, the search for alternative elements to the substrates, became to necessary reduce spends the production. Besides, it's important to quantify the physics - hydric characteristics and the manner to establish the calendar of the irrigations using these materials. Based in this, this research had as the mainly purpose to evaluate the performance and the water consumption of the Kalanchoe blossfeldiana Poelln, $c v$. Gold Jewel in ten alternative substrates and to define the most indicated to the commercial production in environmental conditions of the west- central of the Rio Grande do Sul State. It was tested substrates composed by different proportions of rice shell, ash of rice shell, decomposed organic substance, cattle and poultry manure. The research was accomplished in greenhouse, in a complete randomized experiment, with four replications. In the end of the period it was evaluated: the height, the number of leaves, the number of inflorescence, the number of flowers in each inflorescence and the plants' canopy diameter, in the different treatments. By the results it can be concluded that, to the production of the plants substrates more indicated are $100 \%$ in organic substance volume and $50 \%$ in organic substance volume $+50 \%$ in cattle manure.

Key words: irrigation, water balance, forune-flower.

\section{INTRODUÇÃO}

O cultivo de flores e plantas ornamentais como atividade econômica vem se intensificando ao longo dos anos e está entre as atividades que apresentam, no agronegócio, o maior índice de crescimento, promovendo uma rápida inclusão de trabalhadores no mercado e atuando como multiplicador de empregos (TOMÉ, 2004). O Rio Grande do Sul é um dos maiores produtores de flores e plantas ornamentais do país (KIYUNA et al., 2004), sendo também o terceiro maior consumidor (CUNHA, 2002).

Na produção de flores, de acordo com a finalidade de seu uso, estas podem ser produzidas na

IPrograma de Pós-graduação em Geomática, Universidade Federal de Santa Maria (UFSM), Santa Maria, RS, Brasil.

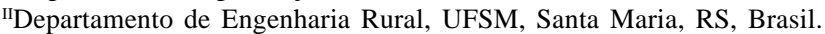

IIIPrograma de Pós-graduação em Engenharia Agrícola, UFSM, 97105-900, Santa Maria, RS, Brasil. E-mail: fatimacibele@yahoo.com.br. Autor para correspondência. 
forma de flores de corte, como mudas ou, ainda, como cultivo em vasos. A vantagem do uso dos vasos encontra-se: 1) na durabilidade das plantas cultivadas desse modo; 2) na versatilidade do seu uso, pois pode ser aplicado em ambientes interiores e exteriores e 3) na facilidade de manejo, evitando, em muitos casos, a manipulação de substratos e as dificuldades do período pós-transplante (MELLO, 2006). Por esses motivos, cada vez mais o mercado consumidor demonstra preferência por flores cultivadas em vaso. Segundo IBRAFLOR (2008), as plantas em vaso de maior procura no mercado consumidor são: crisântemo, violeta e kalanchoe, em função de seu custo de produção, durabilidade das florações e efeito ornamental.

A busca de alternativas para os principais gargalos do processo produtivo de flores de vaso tem como um dos seus principais itens a necessidade de avaliação de formulações para substratos que ofereçam baixo custo de produção e características físicas e químicas que permitam o pleno desenvolvimento da espécie. De acordo com MELLO (2006), a maior parte dos substratos utilizados na floricultura em vaso provêm das regiões Sudeste e Norte do Brasil, podendo, assim, o Rio Grande do Sul duplicar seu custo final em relação a esses Estados.

Dentre os itens que são considerados para viabilização econômica da produção de flores em vaso, em cadeias regionais, pode-se destacar a utilização de misturas com produtos locais para fabricação de substratos. No caso do Estado do Rio Grande do Sul, a casca do arroz é uma alternativa de material, visto que a orizicultura irrigada é responsável por $60,2 \%$ da produção nacional de arroz, sendo o Rio Grande do Sul o maior Estado produtor brasileiro (IBGE, 2009). De acordo com (KÄMPF et al., 2006), a casca de arroz, quando carbonizada, pode resultar em um complemento de substrato que permite o aumento da retenção de água. Além da casca de arroz carbonizada (CAC), destacam-se também as suas cinzas (CZ) como materiais que podem ser obtidos com baixo custo.

A necessidade de se caracterizarem produtos encontrados nas diferentes regiões do país e torná-los disponíveis como substratos agrícolas é fundamental para reduzir os custos de produção (ANDRIOLO et al.,1999). Além disso, a questão ambiental deve ser considerada na escolha dessas matérias-primas para produção de substratos. A disponibilização como substrato agrícola implica a sua caracterização física e química. De acordo com MARTINEZ (2002), dentre as propriedades de um substrato, deve-se destacar a boa retenção de água, na faixa de 1 a $5 \mathrm{kPa}$, a densidade seca, a porosidade total, o espaço de aeração e a água disponível.

A resposta das plantas à disponibilidade de água no substrato tem sido estudada como uma forma de controle da irrigação, já que irrigações deficitárias refletem diretamente na redução da produtividade, enquanto que irrigações excessivas prejudicam a qualidade das flores (FARIAS, 2005). No caso do cultivo de Kalanchoe em vaso, em ambiente protegido, a irrigação é prática fundamental, porém seu manejo é distinguível em função das características físicas do substrato e do tamanho do vaso utilizado no cultivo, e o uso da irrigação implica ganhos adicionais advindos de melhores colheitas. Para não inviabilizar o ganho, a quantidade de água tem que ser adequada, não podendo haver excesso nem falta (FARIAS, 2005).

Com base nessas premissas, este trabalho teve como objetivo avaliar o desempenho e o consumo de água em 10 substratos alternativos, para cultivo em vaso de Kalanchoe blossfeldiana Poelln., cultivar 'Gold Jewel', nas condições ambientais da região centro-oeste do Estado do Rio Grande do Sul.

\section{MATERIAL E MÉTODOS}

O experimento foi conduzido em área experimental da Universidade Regional Integrada do Alto Uruguai e das Missões, em Santiago Rio Grande do Sul (RS), em estufa com sistema de refrigeração do tipo Pad \& Fan Os materiais componentes dos substratos foram: casca de arroz carbonizada, cinza de casca de arroz, esterco bovino, esterco avícola e matéria orgânica doméstica, gerada a partir da decomposição de lixo orgânico doméstico.

$\mathrm{O}$ experimento foi conduzido em delineamento experimental inteiramente casualizado, com 10 tratamentos e sete repetições. Os tratamentos constituíram de diferentes substratos testados para a cultivar 'Gold Jewel de Kalanchoe', compostos por substâncias puras e misturadas utilizando-se o critério volume por volume, nas seguintes proporções: a) materiais puros: $\mathrm{T} 1(\mathrm{CAC})=100 \%$ de casca de arroz carbonizada, $\mathrm{T} 2(\mathrm{CZ})=100 \%$ de cinza de casca de arroz, T3 (EB) $=100 \%$ de esterco bovino e T4 (MO)=100\% de matéria orgânica; e b) misturas: T5 (CAC+EB)=60\% de casca de arroz carbonizada $+40 \%$ de esterco bovino, T6 (MO+EA)=70\% de matéria orgânica $+30 \%$ de esterco avícola, $\mathrm{T} 7(\mathrm{CZ}+\mathrm{EB})=60 \%$ de cinza de casca de arroz + $40 \%$ de esterco bovino, $\mathrm{T} 8(\mathrm{CAC}+\mathrm{EA})=70 \%$ de casca 
de arroz carbonizada $+30 \%$ de esterco avícola, T9 $(\mathrm{CAC}+\mathrm{MO})=50 \%$ de casca de arroz carbonizada $+50 \%$ de matéria orgânica e T10 (MO+EB)=50\% de matéria orgânica $+50 \%$ de esterco bovino. Foi realizada a caracterização física dos diferentes substratos pelas análises: densidade do substrato (DS), relação poros/ sólidos (P/S), espaço de aeração (EA) e retenção de água (CRA). As quantificações foram desenvolvidas de acordo com metodologia proposta por KÄMPF (2006).

Para a produção das mudas, foi utilizado o processo de estaquia. As estacas foram colocadas em bandejas de isopor alveolado, com substrato comercial, com pH variando entre 5,5 e 6,0 e espaço de aeração de aproximadamente de $10 \%$.

O consumo de água da cultura foi determinado por meio da equação do balanço hídrico conforme a seguinte expressão:

$$
\text { Etr }=\sum_{i=1}^{L} M_{i}-\sum_{i=1}^{L} M_{i+1}+I-D, \text { em que Etr é a }
$$

evapotranspiração real da planta em vaso, em um intervalo de tempo $\Delta \mathrm{t}$ de três dias; $\mathrm{M}_{\mathrm{i}}$ é a massa de substrato e água contida no vaso, no início do intervalo de tempo $(\Delta \mathrm{t})$ considerado; i é o índice representando o intervalo de tempo $(\Delta \mathrm{t})$ considerado para o balanço; $\mathrm{M}_{\mathrm{i}+1}$ é a massa de substrato e água remanescente no final do intervalo de tempo $(\Delta \mathrm{t})$ considerado; I é a irrigação aplicada no vaso, no intervalo de tempo $\Delta \mathrm{t}$, e $\mathrm{D}$ é a percolação (ou drenagem). A variação do armazenamento de água no vaso $\left(\mathrm{M}_{\mathrm{i}}-\mathrm{M}_{\mathrm{i}+1}\right)$ ocorreu por meio da pesagem dos vasos em uma balança com precisão de 0,001g. Foi realizada a fertirrigação em período quinzenal, com 60g Kristalon, fertilizante composto por nutrientes essenciais à planta, diluídos em 100 litros de água. A irrigação e a fertirrigação foram realizadas manualmente, com o auxílio de uma proveta.

Em função do crescimento vegetativo, foi avaliada a evolução da biomassa seca do sistema radicular (g) da cultivar, por meio da retirada da planta inteira do vaso, sem danificar as raízes. Efetuou-se a avaliação em um vaso de cada tratamento, em três períodos distintos do desenvolvimento da cultura, aos 30 dias após transplantio (DAT), 50DAT e 70DAT. Para analisar as características da cultivar, foram avaliadas as plantas nos diferentes tratamentos, no período de floração (ponto de comercialização). As variáveis analisadas como componentes finais de rendimento foram: altura de planta $(\mathrm{cm})$, número de folhas, número de inflorescências, número de flores e diâmetro da copa das plantas (cm). Os dados obtidos foram analisados via análise de variância, e as médias foram submetidas ao teste Tukey e à análise de regressão polinomial, com 5\% de probabilidade de erro.

\section{RESULTADOS E DISCUSSÃO}

Na tabela 1, são apresentados os resultados das análises físicas realizadas nos diferentes tratamentos. Observa-se que os menores valores de densidade de substrato foram apresentados no tratamento de CAC, com 213,6g L-1. Segundo BUNT (1973), a densidade do substrato é considerada ideal para o pleno desenvolvimento da planta, entre 400 e

Tabela 1 - Características físicas dos materiais e das misturas formuladas com a finalidade de uso como substrato para a produção de Kalanchoe blossfeldiana, cultivar 'Gold Jewel', em vaso.

\begin{tabular}{lcccc}
\hline Tratamentos & $\mathrm{DS}^{1}\left(\mathrm{~g} \mathrm{l}^{-1}\right)$ & EA (\%) & CRA (ml) & P/S (\%) \\
\hline CAC & 213,6 & 25,0 & 502,0 & 3,0 \\
CZ & 228,7 & 26,0 & 860,0 & 1,5 \\
EB & 739,3 & 4,0 & 413,0 & 0,6 \\
MO & 935,2 & 7,0 & 574,0 & 1,0 \\
CAC+EB & 451,2 & 29,0 & 261,0 & 1,2 \\
MO+EA & 836,3 & 3,0 & 428,0 & 0,7 \\
CZ+EB & 575,0 & 8,0 & 627,0 & 1,2 \\
CAC+EA & 233,6 & 33,0 & 371,0 & 2,3 \\
CAC+MO & 479,0 & 17,0 & 425,0 & 1,5 \\
MO+EB & 860,5 & 19,0 & 400,0 & 0,3 \\
\hline
\end{tabular}

${ }^{1} \mathrm{DS}=$ densidade do substrato; EA=espaço de aeração; CRA=capacidade de retenção de água; P/S=relação volume de poros/volume de sólidos. 
$500 \mathrm{~g} \mathrm{~L}^{-1}$, sendo assim os tratamentos com CAC, CZ e CAC+EA não apresentam densidade suficiente para garantir a estabilidade do recipiente, por apresentarem valores inferiores ao ideal, assim como os tratamentos com substratos de EB, MO, MO+EA e MO+EB também não apresentaram densidade satisfatória, por apresentarem valores superiores ao recomendado. Os tratamentos com CAC, CZ, CAC+EB e CAC+EA apresentaram os maiores espaços de aeração, ideais para o desenvolvimento da planta. Segundo PENNINGSFELD (1983), o espaço ideal é próximo a 30\%.

Observou-se que os tratamentos com substratos de CZ e CZ+EB apresentaram os maiores valores para a capacidade de retenção de água. KÄMPF (2006) relata a importância dessa característica na frequência de irrigação, uma vez que esta depende da presença e do tamanho dos poros do substrato. Segundo a mesma autora, a relação P/S das matériasprimas indicadas para uso na composição de substratos deve-se encontrar geralmente acima de 3,0\%, verificando-se que somente o tratamento de CAC tem uma boa relação volume de poros-volume de sólidos.

A cultivar 'Gold Jewel' apresentou diferença no consumo médio diário de água entre os substratos analisados, em consequência destes apresentaram características físico-hídricas distintas (Tabela 2). Observa-se que o tratamento que apresentou o maior consumo médio de água foi o substrato CAC, 3,06mm, diferindo apenas do tratamento com substrato de $\mathrm{CAC}+\mathrm{MO}$, e este apresentou o menor consumo hídrico médio diário, 2,80mm. Os resultados encontrados estão de acordo com SOARES et al. (2008), os quais, testando diversas lâminas de irrigação, encontraram um consumo médio diário máximo e mínimo de 3,4 e 1,9mm, respectivamente, para a cultivar 'Gold Jewel', transplantada no mês de fevereiro. Esses resultados também são coerentes com REICHARDT (1990), que reporta a direta relação das condições climáticas em que as plantas estão submetidas com o processo de evapotranspiração destas. TJOSVOLD \& SCHULBACH (1991), em estudo com flores de corte, examinando alternativas apropriadas para um maior controle da irrigação, relatam que a irrigação é fundamental para o cultivo em ambiente protegido, porém seu manejo adequado tem sido negligenciado pelos produtores, resultando em prejuízos no crescimento vegetal e consequentes decréscimos na produtividade e na qualidade do produto final. Esses mesmos autores encontraram cultivos em que normalmente a irrigação não é frequente e, quando feita, é aplicada em demasia. Além disso, PEREIRA et al. (2009), por meio de experimento em que foram testados quatro níveis de tensão de água no solo (15, 25, 40 e $60 \mathrm{kPa}$ ), no cultivo de gladíolo, destacam a importância da irrigação no cultivo de plantas ornamentais. Esses autores observaram que a altura da planta, o tamanho da haste floral e o número de

Tabela 2 - Valores médios do consumo diário de água e dos caracteres agronômicos para a cultivar 'Gold Jewel’, nos diferentes substratos testados.

\begin{tabular}{|c|c|c|c|c|c|c|}
\hline \multirow{2}{*}{ Tratamentos } & \multirow{2}{*}{$\begin{array}{l}\text { Consumo de água } \\
\qquad\left(\mathrm{mm} \mathrm{dia}^{-1}\right)\end{array}$} & \multirow{2}{*}{ Altura (cm) } & \multicolumn{3}{|c|}{--------------------Número Final------------------- } & \multirow{2}{*}{$\begin{array}{l}\text { Diâmetro Copada } \\
\text { (cm) }\end{array}$} \\
\hline & & & Folhas & Inflorescências & Flores & \\
\hline CAC & $3.04 \mathrm{a}^{1}$ & 15,5 bc & 50,0 bc & $8,2 \mathrm{abc}$ & $62,5 \mathrm{~cd}$ & 9,7 def \\
\hline $\mathrm{CZ}$ & $2.91 \mathrm{ab}$ & 11,7 c & $32,7 \mathrm{c}$ & 12,5 a & $40,5 \mathrm{~d}$ & $8,1 \mathrm{f}$ \\
\hline EB & $3.00 \mathrm{ab}$ & 19,5 a & $73,5 \mathrm{ab}$ & $5,0 \mathrm{c}$ & $164,7 \mathrm{ab}$ & 14,4 a \\
\hline MO & $2.92 \mathrm{ab}$ & $18,6 \mathrm{ab}$ & $59,5 \mathrm{abc}$ & 6,5 bc & 112,0 bcd & 10,7 bcde \\
\hline $\mathrm{CAC}+\mathrm{EB}$ & $2.95 \mathrm{ab}$ & $18,7 \mathrm{ab}$ & $66,7 \mathrm{ab}$ & $9,5 \mathrm{abc}$ & 137,0 bcd & $12,1 \mathrm{bc}$ \\
\hline $\mathrm{MO}+\mathrm{EA}$ & $2.87 \mathrm{ab}$ & $17,2 \mathrm{ab}$ & $66,5 \mathrm{ab}$ & 9,25 abc & 113,5 bcd & 11,7 bcd \\
\hline $\mathrm{CZ}+\mathrm{EB}$ & $2.99 \mathrm{ab}$ & $18,4 \mathrm{ab}$ & $66,5 \mathrm{ab}$ & 12,5 a & 194,2 a & 11,7 bcd \\
\hline CAC+EA & $2.94 \mathrm{ab}$ & $19,0 \mathrm{ab}$ & 78,0 a & $11,0 \mathrm{ab}$ & $156,0 \mathrm{ab}$ & $12,6 \mathrm{ab}$ \\
\hline $\mathrm{CAC}+\mathrm{MO}$ & $2.80 \mathrm{~b}$ & $18,1 \mathrm{ab}$ & 58,5 abc & 5,5 c & $73,5 \mathrm{~cd}$ & 9,3 ef \\
\hline $\mathrm{MO}+\mathrm{EB}$ & $3.06 \mathrm{a}$ & $19,0 \mathrm{ab}$ & $66,5 \mathrm{ab}$ & 6,5 bc & 114,7 bcd & 10,3 cde \\
\hline Média & 2.95 & 17,6 & 61,8 & 8,6 & 116,9 & 11,1 \\
\hline CV\% & 3.95 & 9,1 & 18,3 & 24,7 & 26,5 & 8,1 \\
\hline DMS \% & 9.52 & 21,9 & 44,2 & 59,6 & 11,3 & 19,5 \\
\hline
\end{tabular}

${ }^{1}$ Médias não seguidas pela mesma letra, na coluna, diferem estatisticamente pelo teste de Tukey, a 5\% de probabilidade de erro. 
flores foram significativamente reduzidos pelo aumento dos níveis de tensão de água no solo e que os melhores resultados em relação ao tamanho da planta, da haste floral e do número de flores foram obtidos mantendose a tensão de água no solo próxima à capacidade de campo (15kPa).

Em razão das diferenças das propriedades físico-hídricas entre os substratos estudados, observou-se variação quanto às variáveis que compõem a produção final do Kalanchoe (Tabela 2). Nota-se que as plantas transplantadas nos substratos de CAC, CZ, CAC+EA e CAC+EB destacam-se com a melhor classificação comercial, apresentando plantas copadas, com grande número de folhas e inflorescências. SILVA \& VISCONTI (1991) descrevem que um bom substrato deve apresentar boa capacidade de retenção de nutrientes e umidade, boa aeração, baixa resistência à penetração das raízes e boa resistência à perda de estrutura.

O substrato que apresentou o maior número médio de folhas foi CAC+EA, e este apresenta espaço de aeração ideal para o desenvolvimento da planta, diferenciando-se dos substratos CAC e CZ. O maior número de inflorescências por planta foi observado nos substratos CZ e CZ+EB, e esse aumento pode estar relacionado com a capacidade de retenção de água, uma vez que estes apresentaram valores satisfatórios para esse parâmetro físico (KÄMPF, 2006). Quanto ao número de flores por planta, o substrato $\mathrm{CZ}+\mathrm{EB}$ apresentou o maior valor, em razão de apresentar condições ideais de retenção de água. Segundo LESSA et al. (2009), para espécies de Kalanchoe, apenas uma dessas variáveis não pode ser levada em consideração por si só para determinar seu aspecto ornamental, mas sim o conjunto destas é que atenderão o padrão comercial exigido.

Esses resultados foram semelhantes aos encontrados por MELLO (2006), que, ao testar diferentes substratos no cultivo de lírio asiático, descobriu que, quando essa espécie é conduzida em vaso, o substrato com $50 \%$ em volume de cinza de casca de arroz carbonizada com a combinação substrato de fibra de coco ou substrato terra do paraíso (formulado com casca de pinus triturada, vermiculita e areia) apresentou maior número de inflorescência e que mais rapidamente atingiu o ponto de comercialização. Além disso, essa mesma autora relata a relação direta entre os componentes de produção da cultura e as características físico-hídricas dos substratos. Esses resultados ainda estão de acordo com os encontrados por SOARES et al. (2006), os quais, trabalhando com diferentes lâminas de irrigação na cultivar 'Debbie', encontraram resultados semelhantes para os mesmos componentes avaliados.

A evolução da biomassa seca do sistema radicular do Kalanchoe é apresentada na figura 1, na qual se observa que todos os tratamentos tiveram um comportamento semelhante, com coeficientes de correlação ajustados a partir de uma curva de resposta linear, apresentando valores elevados $r^{2}=0,9996$ (máximo) e r²=0,7069 (mínimo). Nota-se que, para quase todos os tratamentos, houve similaridade no crescimento, com aumento do sistema radicular com o passar dos DAT, com exceção do tratamento com substrato MO + EA, que, aos 50DAT, diminuiu seu sistema radicular em relação à primeira avaliação (30DAT). Verifica-se que o maior peso de biomassa (g) aos 30 e 70DAT foi observado no tratamento com substrato de $\mathrm{CZ}+\mathrm{EB}$, enquanto que, aos 50DAT, o maior peso (g) foi no substrato de CAC. Esses resultados discordam dos encontrados por GONÇALVES \& MINAMI (1994), os quais, analisando o efeito do substrato artificial no enraizamento de estacas de kalanchoe (Kalanchoe blossfeldiana, cultivar 'Singapur'), verificaram que as misturas que continham casca de arroz tostada apresentaram redução no volume de raízes. Além disso, STUMPF et al. (2001), testando substratos de vermiculita fina e casca de arroz carbonizada, observaram que o susbtrato com vermiculita fina proporcionou maior desenvolvimento do sistema radicular de estacas de Chamaecyparis lawsoniana, tanto em comprimento, como em volume, em razão da grande capacidade de aeração e retenção de água.

\section{CONCLUSÃO}

Os substratos mais indicados para a produção comercial de Kalanchoe blossfeldina Poelln, nas condições ambientais da região centro-oeste do Estado do Rio Grande do Sul, são: 60\% de cinza de casca de arroz $+40 \%$ de esterco bovino e $70 \%$ de casca de arroz carbonizada $+40 \%$ esterco avícola, com um consumo médio diário de água de 2,99 e 2,94mm, respectivamente.

Ciência Rural, v.40, n.3, mar, 2010. 

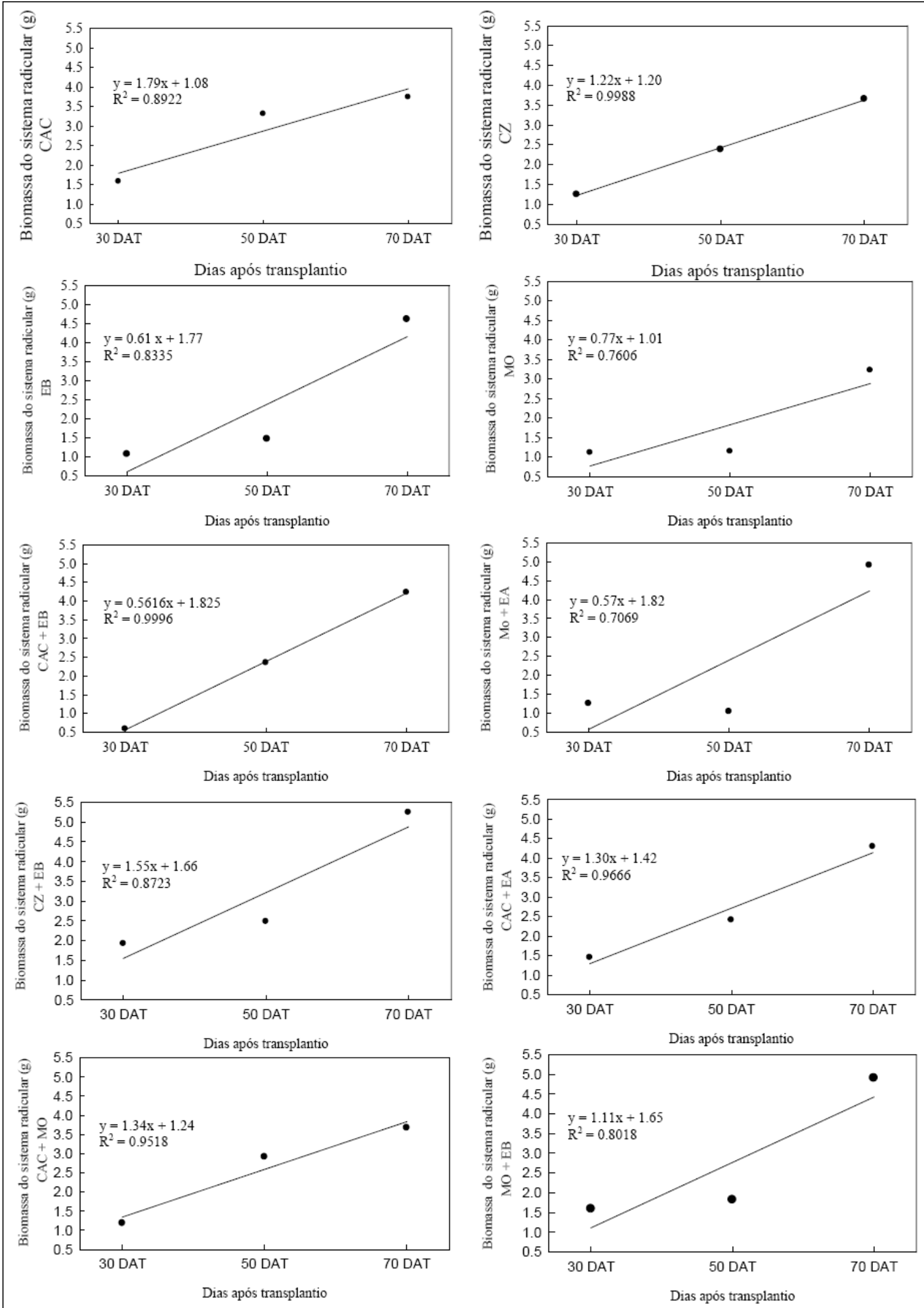

Figura 1 - Biomassa seca (g) do sistema radicular para a cultivar 'Gold Jewel’ em épocas distintas de avaliação, nos diferentes substratos. 


\section{REFERÊNCIAS}

ANDRIOLO, J.L. et al. Caracterização e avaliação de substratos para o cultivo do tomateiro fora do solo. Horticultura brasileira, Campinas, v.17, n.3, p.215-220, 1999. Disponível em: <http://www.abhorticultura.com.br/biblioteca/arquivos/ Download/biblioteca/hb_17_3.pdf\#page=37>. Acesso em: 20 de set. 2009.

BUNT, A.C. Some physical and chemical characteristics of loamless pot-plant substrates and their relation to plant growth. Plant and Soil, Austrália, v.1, n.38, p.1954- 1965, 1973.

CUNHA, D.R. Os fatores críticos de sucesso do comércio flores e plantas ornamentais: as lojas de arte floral no município Alegre/RS. 2002. 115f. Dissertação (Mestrado em Administração) Universidade Federal do Rio Grande do Sul, Porto Alegre, RS.

FARIAS, M.F.; SAAD, J.C. Crescimento e qualidade de crisântemo cultivado em vaso sob ambiente protegido. Horticultura. Brasileira, Campinas, v.23, n.3, p.740-742. 2005. Disponível em: < http:// www.scielo.br/scielo.php?script=sci_arttext\&pid=S010205362005000300010>. Acesso em: 12 de set. 2009. doi: 10.1590/ S0102-05362005000300010.

GONÇALVES, A.L.; MINAMI, K. Efeito de substrato artificial no enraizamento de estacas de calanchoe ( Kalanchoe $\mathrm{x}$ blossfeldiana cv. 'Singapur', Crassulaceae). Revista Scientia Agrícola, Piracicaba, v.51, n.2, p.240-244, 1994. Disponível em: <http://www.scielo.br/pdf/sa/v51n2/07.pdf>. Acesso em: 20 de set. 2009.

INSTITUTO BRASILEIRO DE FLORICULTURA IBRAFLOR. Floricultura no Brasil. Campinas, SP. 25 de Nov. Acessado em 15 de nov. 2008. Online. Disponível em: $<$ http://www.ibraflor.org/sis.interna.asp?pasta=1\&pagina=90>.

INSTITUTO BRASILEIRO DE GEOGRAFIA E ESTATÍSTICA. Ministério do planejamento, orçamento e gestão. Brasil 23 de mar. Acessado em 23 de mar de 2009. Online. Disponível em: <http://www.ibge.gov.br/home/ estatistica/indicadores/agropecuaria/lspa/default.shtm>.

KÄMPF, A.N. et al. Floricultura: técnicas de preparo de substratos. Brasília: LK, 2006. 132p.

KIYUNA, K. et al. Floricultura brasileira no início do século XXI: o perfil do produtor. Informações Econômicas, São Paulo, v.34, n.4, p.14-32, 2004. . Disponível em: <http:// www.iea.sp.gov.br/OUT/publicacoes/pdf/tec2-0404.pdf $>$. Acessado em 15 de nov. 2008.

LESSA, M.A. et al. Application of different fertilizers in substrate for Kalanchoe luciae Raym.-Hamet cultivation. Ciência e Agrotecnologia, Lavras, v.33, n.4, p.950-955, 2009. Disponível em: <http://www.scielo.br/pdf/cagro/v33n4/ a02v33n4.pdf>. Acesso em: 2009.

MARTINEZ, P.F. Manejo de substratos para horticultura. In: ENCONTRO NACIONAL DE SUBSTRATOS PARA
PLANTAS: CARACTERIZAÇÃO, MANEJO E QUALIDADE DE SUBSTRATOS PARA PRODUÇÃO DE PLANTAS, 2002, Campinas, SP. Anais... Campinas: IAC, 2002. p.53-76.

MELLO, R.P. Consumo de água do lírio asiático em vaso com diferentes substratos. 2006. 74f. Dissertação (Mestrado em Engenharia Agrícola) - Universidade Federal de Santa Maria, Santa Maria, RS.

PENNINGSFELD, F. Kultursubstrate fur den gartenbau, besonders in Deutschland: ein kritischer Überblick. Plant and Soil, The Hague, v.75, n, 2, p.269-281, 1983. Disponível em: <http://www.springerlink.com/content/e351721104g22614/ fulltext.pdf $>$. Acesso em: 20 de set. 2009. doi: 10.1007/ BF02369967.

PEREIRA, J.R.D. et al. Crescimento e produção de hastes florais de gladíolo cultivado sob diferentes tensões de água no solo. Ciência e Agrotecnologia, Lavras, v.33, n.4, p.965-970, 2009. Disponível em: <http://www.scielo.br/scielo.php?pid=S141370542009000400004\&script=sci_arttext $>$. Acesso em: 20 de set. 2009. doi: 10.1590/S1413-705420090004000 04 .

REICHARDT, K. A água em sistemas agrícolas. Piracicaba: Manole, 1990. 188p.

SILVA Jr, A.A.; VISCONTI, A. Recipientes e substratos para a produção de mudas de tomate. Agropecuário Catarinense, Florianópolis, v.4, n. 4, p.20-23, 1991. Disponível em: <http:/ / w w w. s c i el o.br/s c i e lo.p h p ? p i d = S 0102 5362004000300023\&script $=$ sci_abstract\&tlng=e $>$. Acesso em: 20 de set. 2009 doi: 10.1590/S0102-05362004000300023.

SOARES, F.C. et al. Resposta de Kalanchoe Blossfeldiana cultivado em diferentes épocas de transplante e submetida a diferentes estratégias de irrigação. In: SEMINÁRIODE INICIAÇÃO CIENTIFICA,9., 2006, Santiago. Anais... Santiago: Universidade Regional Integrada do Alto Uruguai e das Missões, 2006. V.1, p.232. CD-Room.

SOARES, F.C. et al. Produtividade sazonal de kalanchoe cultivado em ambiente protegido e submetido a estratégias de irrigação. Revista Irriga, Botucatu, v.13, n.4, p.492-506, 2008.

STUMPF, E.R.T. et al. Efeito do ácido indolbutírico, substrato e tipo de estaca no enraizamento de chamaecyparis lawsoniana Parl. Revista Brasileira de Agrociência, Pelotas v.7 n.2, p.101-105, 2001. Disponível em: <http://www.ufpel.tche.br/ faem/agrociencia/v7n2/artigo05.pdf> . Acesso em: 20 de set. 2009.

TJOSVOLD, S.A.; SCHULBACH, K.F. How to reduce water use and maximize yields in greehnouse roses. California Agriculture, United States of America, v.45, n.3. p.31-32, 1991.

TOMÉ, L.M. Avaliação do desempenho logísticooperacional de empresas no setor da floricultura: um estudo de caso no Ceará. 2004. 163f. Dissertação (Mestrado em Engenharia de Transportes) - Universidade Federal do Ceará, Fortaleza, CE. 